\title{
Zeuxine grandis and Z. chowdheryi (Orchidaceae): New records to Eastern Ghats, peninsular India
}

\author{
T.S. Saravanan and S. Kaliamoorthy* \\ National Orchidarium and Experimental Garden, Botanical Survey of India (BSI), \\ Southern Regional Centre (SRC), Yercaud - 636602, Salem district, Tamil Nadu, India. \\ *E-mail: bsinoegyercaud@gmail.com
}

\begin{abstract}
Zeuxine grandis Seidenf. and Z. chowdheryi Av.Bhattacharjee \& Sabap. (Orchidaceae) are reported here for the first time for Eastern Ghats from Tamil Nadu. Brief descriptions along with photographs are provided to facilitate easy recognition of these species.
\end{abstract}

Keywords: Eastern Ghats, Orchids, Tamil Nadu, Zeuxine grandis, Zeuxine chowdheryi

\section{Introduction}

The genus Zeuxine Lindl. was established by John Lindley (1826) in the appendix to his 'Collectanea Botanica'. It is represented by c. 80 species (Chen et al., 2009), and distributed from tropical Africa, Madagascar through India, Pacific Islands and Sri Lanka (Bose \& Bhattacharjee, 1999; Govaerts et al., 2012). In India, it is represented by 19 species, of which 7 are reported from peninsular India (Misra, 2007).

During the floristic exploration in the Sanyasimalai Reserve Forest, Yercaud, Salem district of Tamil $\mathrm{Nadu}$, we have collected two interesting terrestrial species of Zeuxine. After critical examination of the specimens with pertinent literature (Chen et al., 2009; Bhattacharjee \& Chowdhery, 2010; Bhattacharjee et al., 2011; Das \& Gogoi, 2013), these were identified as $Z$. grandis Seidenf. and Z. chowdheryi Av.Bhattacharjee \& Sabap. (Bhattacharjee \& Sabapathy, 2010). Though these two species of Zeuxine are reported from Western Ghats (Sasidharan et al., 1999; Manickam et al., 2003; Bhattacharjee \& Sabapathy, 2010), but hitherto not reported from the Eastern Ghats (Matthew, 1983;Ansari\&Dwarakan,1995;Dwarakan \& Ansari, 2001; Krishna Swamy et al., 2004; Rao \& Sridhar, 2007; Reddy et al., 2008; Karuppusamy et al., 2009; Rajarajeswari \& Nandakumar, 2015). Therefore, the present collections from Sanyasimalai Reserve Forest constitute new distributional records of Z. grandis and Z. chowdheryi for Eastern Ghats of peninsular India.

Zeuxine grandis Seidenf., Dansk Bot. Ark. 32(2): 90, t. 56. 1978; Aver., Turczaninowia 11(1): 154. 2008; Av.Bhattacharjee \& H.J. Chowdhery, Taiwania 55(3): 315. 2010; Av.Bhattacharjee et al.,
Richardiana 11(2): 98. 2011; R. Das \& K. Gogoi, McAllen Int. Orchid Soc. J. 14(1): 9. $2013 . \quad$ Fig. 1

Herb, terrestrial, rhizomatous, 18.7-20.3 cm long, slender. Rhizomes creeping, rooting at nodes, c. $3.5 \times$ $1.8 \mathrm{~cm}$, glabrous, greenish brown. Stems erect, terete, 6.5-8.9 × 1.5-2.3 cm, glabrous, greenish brown. Leaves 4 or 5 , subrosulate, greenish brown; petioles $c$. $0.2 \mathrm{~cm}$ long, sheathing at base, sheaths membranous; lamina ovate-lanceolate, margins entire, apex acute, c. $2.7 \times 1.3 \mathrm{~cm}$, pendulous and withered during flowering, often reddish. Inflorescence lax spike, 4 or 5-flowered; spikes terminal, erect; peduncles terete, $7.5-9.8 \mathrm{~cm}$ long with 1 or 2 sterile bracts, pubescent; sterile bracts ovate-lanceolate, c. $0.9 \times 0.3 \mathrm{~cm}$, apex acuminate, pubescent, reddish brown, 3-veined; rachis 3.5-5.2 cm long. Flowers resupinate, 1.2-1.4 cm long; floral bracts ovate-lanceolate, c. $0.7 \times 0.2 \mathrm{~cm}$, apex acuminate, pubescent on margins and lower half of abaxial surface, reddish brown, 1-veined, as long as the ovary. Sepals free, pubescent, brownish green, white towards apex, dorsal sepal broadly ovate, c. $0.6 \times 0.3 \mathrm{~cm}$, concave, apex obtuse, forming a hood with petals, 1-veined; lateral sepals ovatelanceolate, c. $0.4 \times 0.2 \mathrm{~cm}$, apex obtuse, 1-veined. Petals slightly oblique, subrectangular-falcate to narrowly ovate, $c .0 .6 \times 0.2 \mathrm{~cm}$, apex obtuse, glabrous, white with brownish green, 2-veined. Lip white, connate to the column base, $c .1 .1 \mathrm{~cm}$ long, Y-shaped, 3-partite; hypochile concave-saccate, c. 0.2 $\times 0.15 \mathrm{~cm}$, white with yellowish tinge, 1 filiform to oblong appendage in each side; mesochile oblong, c. $0.3 \times 0.15 \mathrm{~cm}$, margins incurved, white; epichile 2-lobed, lobules orbicular-obovate, c. $0.7 \times 0.3$ $\mathrm{cm}$, white with yellow tinge at base, broadening from base, the angle between two lobules usually more acute, margins undulate. Column c. 0.2 

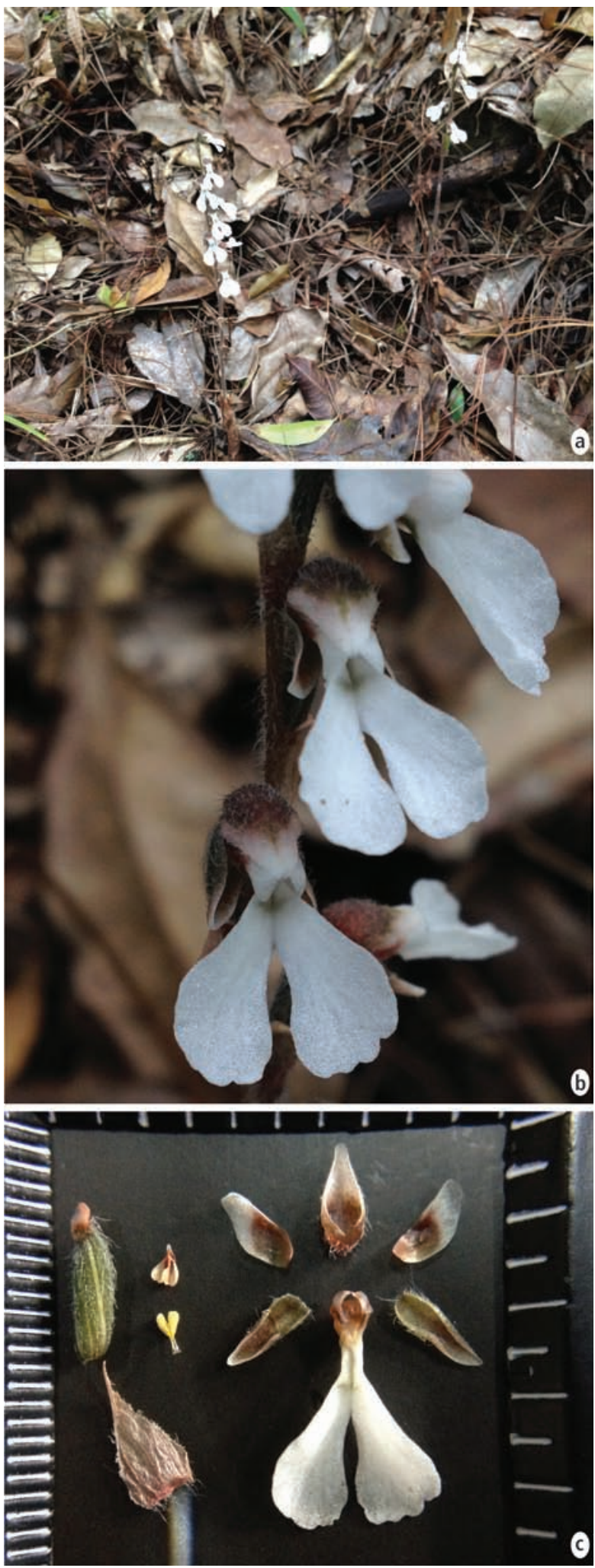

Fig. 1. Zeuxine grandis Seidenf.: a. Habit; b. Inflorescence; c. Perianth parts.

$\mathrm{cm}$, wings triangular; stigma with 2-glandular protuberances at the sides. Pollinarium $c .0 .2 \mathrm{~cm}$, bifid; anthers ovoid-lanceolate, $0.1-0.2 \mathrm{~cm}$ long; pollinia 2, pyriform, yellow; rostellum oblanceolate, c. $0.06 \mathrm{~cm}$ long, slightly curved; tegula linear, semitransparent, greenish yellow; viscidium oblong, thick, transparent, white. Ovary including pedicel fusiform, c. $0.6 \mathrm{~cm}$ long, ridged, pubescent. Capsules ellipsoid, c. $0.6 \mathrm{~cm}$ long, green.

Flowering \& fruiting: January-February.

Habitat: Very rarely found in the shade of trees, rich in humus with leaf litters in dry deciduous to semi evergreen forest, in association with Cheirostylis flabellata (A. Rich.) Wight and Zeuxine longilabris (Lindl.) Trimen, at elevations between 800 and $1480 \mathrm{~m}$.

Distribution: China, India (Andaman and Nicobar Islands, Assam, Odisha, Sikkim, Tamil Nadu and Uttarakhand), Thailand and Vietnam.

Specimen examined: INDIA, Tamil Nadu, Salem district, Sanyasimalai Reserve Forest, Yercaud, $11^{\circ} 47^{\prime} 59^{\prime \prime}$ N, 78 $12^{\prime} 52^{\prime \prime}$ E, \pm 1440 m, 10.1.2017, T.S. Saravanan \& S. Kaliamoorthy 109602 (spirit collection).

Zeuxine chowdheryi Av.Bhattacharjee \& Sabap., Taiwania 55(4): 342. 2010.

Fig. 2

Herb, terrestrial, 17-18.5 cm high. Rhizomes c. 6.4 $\times 1.5 \mathrm{~cm}$, dull pinkish brown, creeping, rooting at nodes on lower side; roots $c .1 .1 \times 0.3 \mathrm{~cm}$, fleshy with distinct root hairs, yellowish brown. Stems erect, $c$. $9.8 \times 1.7 \mathrm{~cm}$, decumbent at base, unbranched with several membranous sheaths formed by withered leaf bases, glabrous, greenish pink. Leaves 3 or 4, withered during flowering, glabrous; petioles $0.3-0.7 \mathrm{~cm}$ long, sheathing at base; lamina ovate to ovate-lanceolate, c. $4.8 \times 1.6 \mathrm{~cm}$, base obtuse, apex acute, 3-veined. Inflorescences terminal racemes, laxly 4-6-flowered, pubescent, pale yellowish brown; peduncles $6.5-7.6 \mathrm{~cm}$ long with 2 or 3 sheathing bracts; sheathing bracts lanceolate, c. $1.3 \times 0.3 \mathrm{~cm}$, apex acuminate, pubescent, pinkish red, 3-veined; rachis c. $4.4 \mathrm{~cm}$ long; floral bracts narrowly lanceolate, $c .0 .7 \times 0.2 \mathrm{~cm}$, apex acuminate, pubescent, pinkish red, usually shorter or as long as ovary, 3-veined. Flowers $1.5-1.8 \mathrm{~cm}$ long, resupinate. Sepals free, pubescent, pinkish red whitish-tinged at apex, 1-veined; dorsal sepal broadly ovate, c. $0.6 \times 0.3 \mathrm{~cm}$, concave, apex obtuse; lateral sepals ovate-lanceolate, $c .0 .6 \times 0.2 \mathrm{~cm}$, apex subacute. Petals broadly elliptic, c. $0.5 \times 0.2 \mathrm{~cm}$, oblique, apex round, glabrous, green, white-tinged at apex with 1-complete and 2-incomplete veins. Lip c. $1.4 \mathrm{~cm}$ long, white, 3-lobed; hypochile c. $0.1 \times$ $0.1 \mathrm{~cm}$, shortly saccate at base with 1 entire, curved appendage in each side, brownish; mesochile oblong, c. $0.4 \times 0.1 \mathrm{~cm}$, margins incurved, whitish green; epichile white, 2-lobuled; lobules falcate, c. $0.9 \times 0.4 \mathrm{~cm}$, more or less parallel to mesochile, margins entire, apex obtuse to rounded. Column $c$. $0.2 \mathrm{~cm}$ long with 1 frontal rectangular appendage 

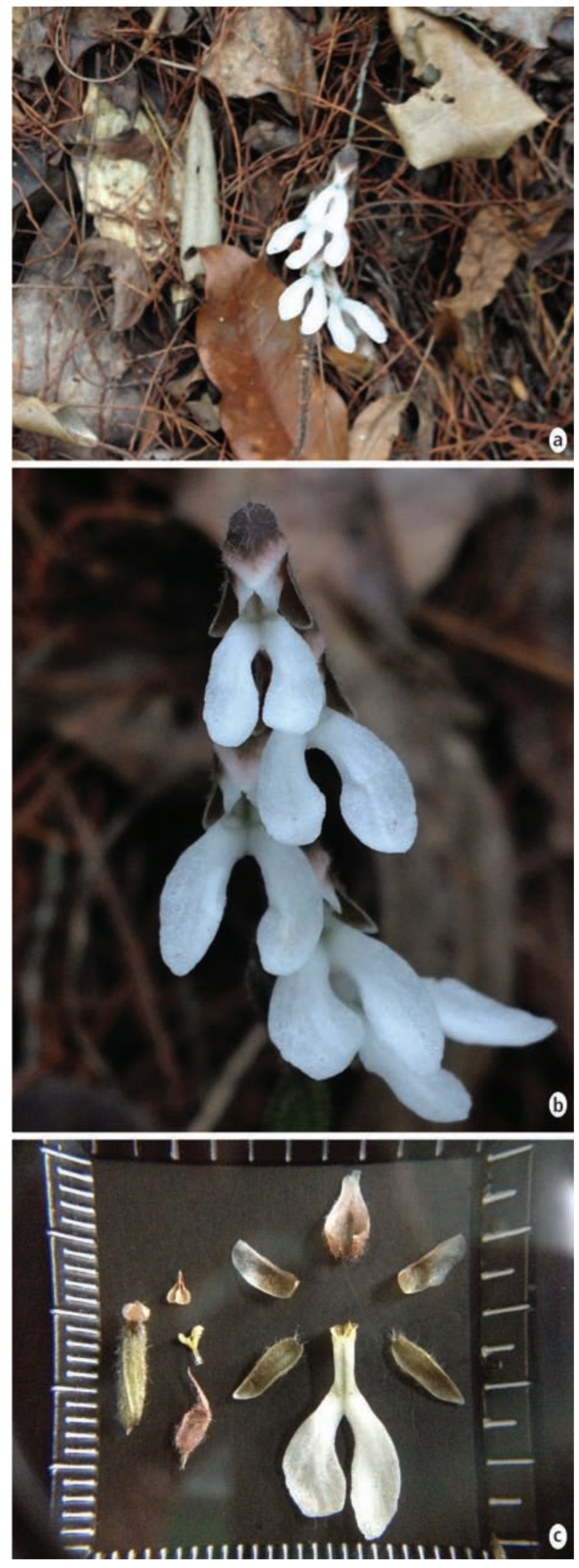

Fig. 2. Zeuxine chowdheryi Av.Bhattacharjee \& Sabap.: a. Habit; b. Inflorescence; c. Perianth parts. on each side near the base of the rostellum; rostellum $0.11-0.14 \mathrm{~cm}$ long, bifid, apex obtuse, slightly curved, dull white. Pollinarium 0.02-0.03 cm long; pollinia 2, c. $0.1 \mathrm{~cm}$ long, sectile, bifid, light yellow to dull white; caudicles $0.03-0.04 \mathrm{~cm}$ long; tegula $0.1-0.2 \mathrm{~cm}$ long, transparent with yellow tinge; viscidium oblong-elliptic, thick, dull white, viscidial end cup-shaped; stigma 2-lobed, suborbicular, well-separated, mustered yellow, laterally placed on column above base of the rostellum. Ovary including pedicel fusiform, c. 0.8 $\times 0.2 \mathrm{~cm}$, pale green, ridged, pubescent. Capsules ellipsoid, c. $1.2 \mathrm{~cm}$ long, green.

Flowering \& fruiting: February-March.

Habitat: Growing under the shade of trees rich in humus with leaf litters in dry deciduous and semievergreen forests, in association with Cheirostylis flabellata (A. Rich.) Wight, Zeuxine grandis Seidenf. and Z. longilabris (Lindl.) Trimen, at elevations between 750 and $1450 \mathrm{~m}$.

Distribution: India: Tamil Nadu (Velliangiri hills, Coimbatore district, Western Ghats; Sanyasimalai Reserve Forest, Yercaud, Salem district, Eastern Ghats). Endemic.

Specimen examined: INDIA, Tamil Nadu: Salem district, Sanyasimalai Reserve Forest, Yercaud, $11^{\circ} 47^{\prime} 59^{\prime \prime} \mathrm{N}, 78^{\circ} 12^{\prime} 52^{\prime \prime} \mathrm{E}, \pm 1447 \mathrm{~m}, 8.2 .2017$, T.S. Saravanan \& S. Kaliamoorthy 109603 (spirit collection).

\section{Acknowledgements}

The authors are thankful to the Director, BSI, and Scientist ' $\mathrm{D}$ ' \& Head of Office, BSI, SRC, Coimbatore, for encouragement and providing facilities. They also thank the Forest Department authorities of Sanyasimalai Reserve Forest, Yercaud, Salem district, Tamil Nadu, for granting permission and guidance in field.

\section{Literature Cited}

Ansari, A.A. \& P. Dwarakan 1995. Studies on the orchids of National Orchidarium and Experimental Garden, Yercaud, Tamil Nadu. J. Econ. Taxon. Bot., Addit. Ser. 11: 129-142.

Bhattacharjee, A. \& H.J. Chowdhery 2010. Zeuxine grandis Seidenf. (Orchidaceae) - An addition to the orchid flora of India. Taiwania 55(3): 315-317.

Bhattacharjee, A. \& C.M. Sabapathy 2010. A new species of Zeuxine Lindl. (Orchidaceae) from India. Taiwania 55(4): 342-345. 
Bhattacharjee, A., Pankaj Kumar, Chowdhery, H.J. \& G.S. Rawat 2011. A new synonym of Zeuxine grandis (Orchidaceae). Richardiana 11(2): 98-103.

Bose, T.K. \& S.K. Bhattacharjee 1999. Orchids of India. Naya Prokash, Calcutta. pp. 1-538.

Chen, S., Gale, S.W., Cribb, P.J. \& P. Ormerod 2009. Zeuxine Lindl. In: Wu, Z.Y., Raven, P.H. \& D.Y. Hong (eds.), Flora of China. Vol. 25 (Orchidaceae). Science Press, Beijing and Missouri Botanical Garden Press, St. Louis. pp.56-57.

Das, R. \& K. Gogoi 2013. Zeuxine grandis Seidenf. (Orchidaceae), an addition to the orchid flora of Assam, India. McAllen Int. Orchid Soc. J. 14(1): 9-12.

Dwarakan, P. \& A.A. Ansari 2001. Addition to the orchids of National Orchidarium and Experimental Garden, Yercaud, Tamil Nadu. J. Econ. Taxon. Bot. 25(3): 539-541.

Govaerts, R., Pfahl, J., Campacci, M.A., Baptista, D.H., Tigges, H., Shaw, J., Cribb, P., George, A., Kreuz, K. \& J. Wood 2012. World Checklist of Orchidaceae. The Board of Trustees of the Royal Botanical Gardens, Kew, published on the Internet: http://www.kew.org/wcsp/ accessed 8 February 2012; 04:20 GMT.

Karuppusamy, S., Muthuraja, G. \& K.M. Rajasekaran 2009. Status of orchids on Kolli hills of Eastern Ghats, Tamil Nadu. EPTRIENVIS Newslett. 15(3): 3-5.

Krishna Swamy, K., Krishna Kumar, H.N., Ramakrishna, T.M. \& S.N. Ramaswamy 2004.
Studies on distribution and phenology of orchids in Karnataka. J. Orchid Soc. India 18(1-2): 81-96.

Manickam, V.S., Uthayakumari, F. \& J. Dulcy 2003. Notes on Zeuxine Lindl. (Orchidaceae) from Tamil Nadu, India. Rheedea 13: 57-62.

Matthew, K.M. 1983. The Flora of the Tamilnadu Carnatic. Vol. 3(2). The Rapinat Herbarium, St. Joseph's College, Tiruchirappalli.

Misra, S. 2007. Orchids of India - A glimpse. Bishen Singh Mahendra Pal Singh, Dehra Dun. pp. $1-402$.

Rajarajeshwari, J. \& K. Nandakumar 2015. Study of orchids diversity in Yercaud Hills, Eastern Ghats, Tamil Nadu. Int. J. Recent Sci. Res. 6(9): 6858-6860.

Rao, T.A. \& S. Sridhar 2007. Wild Orchids in Karnataka (A pictorial compendium). Institute of Natural Resources Conservation, Education, Research and Training, Bangalore. pp. 1-152.

Reddy, C.S., Reddy, K.N. \& V.S. Raju 2008. Supplement to Flora of Andhra Pradesh, India. Deep Publications, New Delhi.

Sasidharan, N., Rajesh, K.P. \& J. Augustine 1999. Zeuxine affinis (Lindl.) Benth. ex Hook.f. (Orchidaceae), a new record for peninsular India. Rheedea 9(2): 159-161.

Received: 30.3 .2017

Revised and Accepted: 14.11.2017 